\title{
iDE: framework inteligente para suporte à decisão: um estudo de caso em EaD
}

\author{
Ketlen K. Teles Lucena ${ }^{1,2}$, Tammy H. Y. Gusmão ${ }^{1}$, \\ Elaine H. Teixeira de Oliveira ${ }^{1}$ \\ ${ }^{1}$ Instituto de Computação - Universidade Federal do Amazonas (UFAM) \\ Manaus, Brasil \\ ${ }^{2}$ Centro de Educação a Distância - Universidade Federal do Amazonas (UFAM) \\ Manaus, Brasil \\ \{ketlen, thyg, elaine\} @icomp.ufam.edu.br
}

\begin{abstract}
Retrieving tacit knowledge and using it as useful information to solve problems requires proper mapping and formalization strategies because it is related to subjective experiences that induce informal but also valuable learning. This paper presents iDE, an intelligent framework that combines AI techniques, such as Case-Based Reasoning (CBR) and Multiagent Systems, with information from a LMS and experts opinions. This combination is used to map implicit knowledge and combine it with explicit knowledge in order to suggest solutions based on past cases. For validation, a case study was proposed covering an educational institution that offers distance learning programs in the Amazon region.
\end{abstract}

Resumo. Recuperar o conhecimento tácito e utilizá-lo como informação útil na resolução de problemas requer estratégias de mapeamento e formalização adequadas, pois trata-se de experiências subjetivas que induzem a um aprendizado informal, porém valioso. Neste artigo é apresentado o iDE, um framework inteligente que combina técnicas de IA, como Raciocínio Baseado em Casos (CBR) e Sistemas Multiagentes, com informações provenientes de um LMS e opiniões de especialistas, para mapear esse conhecimento implícito e combiná-lo ao explícito para sugerir soluções baseadas em casos passados. Para validação foi proposto um estudo de caso abrangendo uma instituição de ensino que oferece cursos na modalidade à distância na região Amazônica.

\section{Introdução}

Mapear o conhecimento tácito com o objetivo de recuperar, formalizar e utilizar suas informações para prevenir, intervir e solucionar problemas constitui um desafio constante em ambientes mutáveis e incertos. Mais especificamente em cenários educacionais, o procedimento de tomada de decisões requer análise e avaliação de históricos e ações anteriores, com base em resultados positivos das estratégias aplicadas [Teles Lucena et al. 2017].

Nesse contexto, é apresentado um framework inteligente que visa auxiliar na solução de problemas, tendo como base o conhecimento adquirido em situações semelhantes a fim de aproveitar resoluções passadas de questões com características equivalentes. Para isso, é discutido um estudo de caso que teve como objeto o Centro de Educação a Distância da Universidade Federal do Amazonas (CED/UFAM).

Instituições que trabalham com Educação a Distância possuem um vasto conhecimento implícito, derivado de experiências dos indivíduos envolvidos em todas as etapas do modelo de ensino-aprendizagem. Esse saber subjetivo resulta em um conjunto de particularidades e características intrínsecas a uma situação específica, identificando- 
VIII Congresso Brasileiro de Informática na Educação (CBIE 2019)

Anais do XXX Simpósio Brasileiro de Informática na Educação (SBIE 2019)

a como única [Teles Lucena et al. 2017]. Administrar o aprendizado à distância - onde o estudante encontra-se em lugar distinto do mediador e o conteúdo é compartilhado de forma assíncrona - exige, além de habilidades gerenciais e pedagógicas, também talento para lidar com as relações nem sempre harmônicas entre os atores do processo. No entanto, esses cursos não enfrentam somente dificuldades de aspectos educacionais, os fatores logísticos e de infraestrutura também figuram como pontos críticos para a gestão deste tipo de ensino [Oliveira et al. 2012].

Normalmente, os dados acadêmicos de um curso à distância ficam armazenados em um Ambiente Virtual de Aprendizagem (AVA ou LMS, do inglês Learning Management System) e as informações de caráter transitório ou temporário não são formalmente descritas, dificultando-se assim o seu reaproveitamento. Esse entendimento não-documentado é acumulado por especialistas que lidam com a realidade do curso, e é utilizado quando alguma situação exige soluções mais complexas e abrangentes [Teles Lucena 2018].

O iDE consiste em um arcabouço conceitual que viabiliza a combinação de técnicas de Inteligência Artificial (IA) - tais como, sistemas multiagentes (MAS) e Raciocínio Baseado em Casos (CBR) - justamente para a recuperação desses conhecimentos explícitos e tácitos envolvidos nas tomadas de decisões comuns a modelos de gestão do ensino à distância. O framework é gerenciado por um sistema multiagente, composto por quatro agentes de software: (i) o agente principal e coordenador dos demais (Manager agent); (ii) o agente que usará a técnica de Raciocínio Baseado em Casos para recuperar e reaproveitar conhecimento de casos passados para de oferecer soluções adaptáveis a novas situações (CBR agent); (iii) o agente que irá identificar e tratar dados acadêmicos provenientes de aplicativos (móveis ou não) inseridos em ambientes virtuais de aprendizagem (LMS agent); e, por último, (iv) o agente responsável por recuperar e formalizar o conhecimento tácito de especialistas e de suas experiências na área de EaD (Experts agent).

O artigo está assim organizado: a Seção 2 traz uma breve revisão da literatura sobre os trabalhos relevantes para esta pesquisa que utilizam CBR e MAS, analisados sob o ponto de vista do problema de adaptação e aproveitamento de conhecimento e experiências anteriores. A Seção 3 apresenta o framework iDE, sua arquitetura e a implementação do sistema multiagente que o caracteriza. Na Seção 4 é apresentado o objeto do estudo de caso, a partir do qual os experimentos de validação do arcabouço foram realizados. A Seção 5 analisa os principais resultados com estes experimentos. As conclusões são discutidas na Seção 6.

\section{Revisão da Literatura}

Nos trabalhos aqui selecionados, explora-se a utilização de recursos de IA, como CBR e MAS, como abordagem de apoio a tomada de decisão - geralmente como suporte a um LMS - fazendo parte de arquiteturas ou frameworks voltados para finalidades educacionais. Ao fim da seção serão avaliadas as contribuições de tais trabalhos para esta pesquisa, visto que nossa proposta se apoia no uso das técnicas de CBR, MAS, e na opinião de especialistas no domínio de cursos na modalidade à distância.

Mendes et al. (2013) apresentaram uma proposta de arquitetura de um agente que engloba todos os mecanismos de um sistema CBR tradicional, como acesso e recuperação de dados, cálculo de similaridade, aprendizado e adaptação de soluções. A base de dados inicial foi formada a partir do processamento da linguagem natural em documentos textuais, sendo que todos os casos foram representados como instâncias de ontologia. 
VIII Congresso Brasileiro de Informática na Educação (CBIE 2019)

Anais do XXX Simpósio Brasileiro de Informática na Educação (SBIE 2019)

Para o agente CBR, todo o mecanismo de inferência depende da análise de casos considerados similares. A autonomia do agente foi caracterizada pelo processo identificação, recuperação e adaptação do caso mais apropriado para a solução do problema proposto. Além disso, o agente proposto foi capaz de absorver a experiência desta solução. A principal contribuição para esta pesquisa foi o uso de uma arquitetura eficiente de agente CBR para reutilização e aprendizado de soluções, utilizando ontologias para formalização do domínio estudado.

$\mathrm{Na}$ proposta de um ITS (Intelligent Tutoring Systems) híbrido do trabalho de Bittencourt et al. (2006) são empregadas técnicas de IA, como CBR e Sistema Baseado em Regras (RBS), e a abordagem de raciocínio é caracterizada por uma arquitetura multiagente. Sua arquitetura apresenta um módulo especialista composto por agentes inteligentes, um módulo de conhecimento de especialistas e uma base de conhecimento modelada por uma ontologia. Os agentes são responsáveis pelas interfaces do módulo e pelo núcleo de raciocínio, que engloba os sistemas CBR e o RBS. O trabalho apresentou uma proposta de combinação de técnicas de IA para melhorar os resultados da recuperação do conhecimento, o que nos inspirou a adotar essa estratégia, acoplando à combinação, a opinião de especialistas e informações de aplicativos de suporte a ambientes virtuais de aprendizagem.

Bremgartner, Netto e Menezes (2014) propuseram uma estratégia para adaptação de conteúdo pedagógico em um LMS a partir de abordagens construtivistas. O trabalho fez uso de MAS e a base de conhecimento dos modelos de aprendizado foi especificada em uma ontologia de domínio. A meta estabelecida foi o desenvolvimento de um modelo focado na aprendizagem direcionada ao indivíduo; esse mesmo modelo sofre adaptações a partir da análise do perfil de cada estudante. A ideia de oferecer ao aluno um modelo individual e personalizado de aprendizagem firmou-se como uma maneira de melhorar o desempenho de estudantes que usam LMS. Os autores afirmam que o uso de MAS associado a uma modelagem do domínio por meio de ontologias pode ser aplicado a outros LMS, uma vez que os agentes e o ambiente de e-learning compartilham o mesmo banco de dados. Essas experiências ajudaram a direcionar a nossa abordagem de análise de informações oriundas do ambiente virtual de aprendizagem.

O trabalho de Garrido et al. (2008) apresenta um modelo para a reutilização de conhecimento por meio da combinação dos conceitos de gestão do conhecimento, CBR e ontologias. Para isso, foi proposta uma construção ontológica de casos para o sistema CBR como um suporte teórico e útil para o compartilhamento e a gestão do conhecimento, de forma que a experiência adquirida em diversas organizações pudesse ser reutilizada nos processos de tomada de decisão. A abordagem desta pesquisa se relaciona a esta porque propôs novos tipos de regras de adaptação estrutural e mecanismos de aprendizado, através da implementação de agentes inteligentes que identificavam casos semelhantes e recuperavam o conhecimento utilizando técnicas de CBR. Assim, a arquitetura multiagente utilizada em conjunto com a construção de uma ontologia para descrição do CBR ampliou o uso desta técnica como meio de gestão do conhecimento, no caso, a memória e a aprendizagem organizacional. $\mathrm{O}$ trabalho concluiu que estas estratégias podem ser utilizadas em organizações, educacionais ou não, para aproveitar as experiências adquiridas em processos decisórios.

As experiências obtidas a partir desses trabalhos contribuíram para nossa pesquisa no sentido de mostrar que o uso de técnicas de IA pode oferecer diversas vantagens quando aplicadas na recuperação e reaproveitamento do conhecimento, em uma realidade de ensino e aprendizagem. 
VIII Congresso Brasileiro de Informática na Educação (CBIE 2019)

Anais do XXX Simpósio Brasileiro de Informática na Educação (SBIE 2019)

\section{O framework iDE}

Esta seção descreve o desenvolvimento do framework iDE, a arquitetura multiagente e as funções de análise, recomendação e aprendizado. O projeto do iDE constitui-se de uma plataforma formada por agentes inteligentes que incorporam características de um sistema distribuído, devido à sua estrutura organizacional no ambiente em que atua. A arquitetura que define o iDE é proposta com base em uma configuração multiagente para acesso semântico ao conhecimento.

A Figura 1 apresenta a arquitetura geral proposta para o framework iDE. Nela pode-se observar a existência de uma interface web para entrada do problema e para a visualização da solução. $\mathrm{O}$ iDE propriamente dito consiste na arquitetura do sistema multiagente, na base de dados que conterá todas as informações cadastradas e geradas, e em uma ontologia que formalizará todas as referências ao domínio do estudo de caso. A seguir, serão descritas cada unidade do framework.

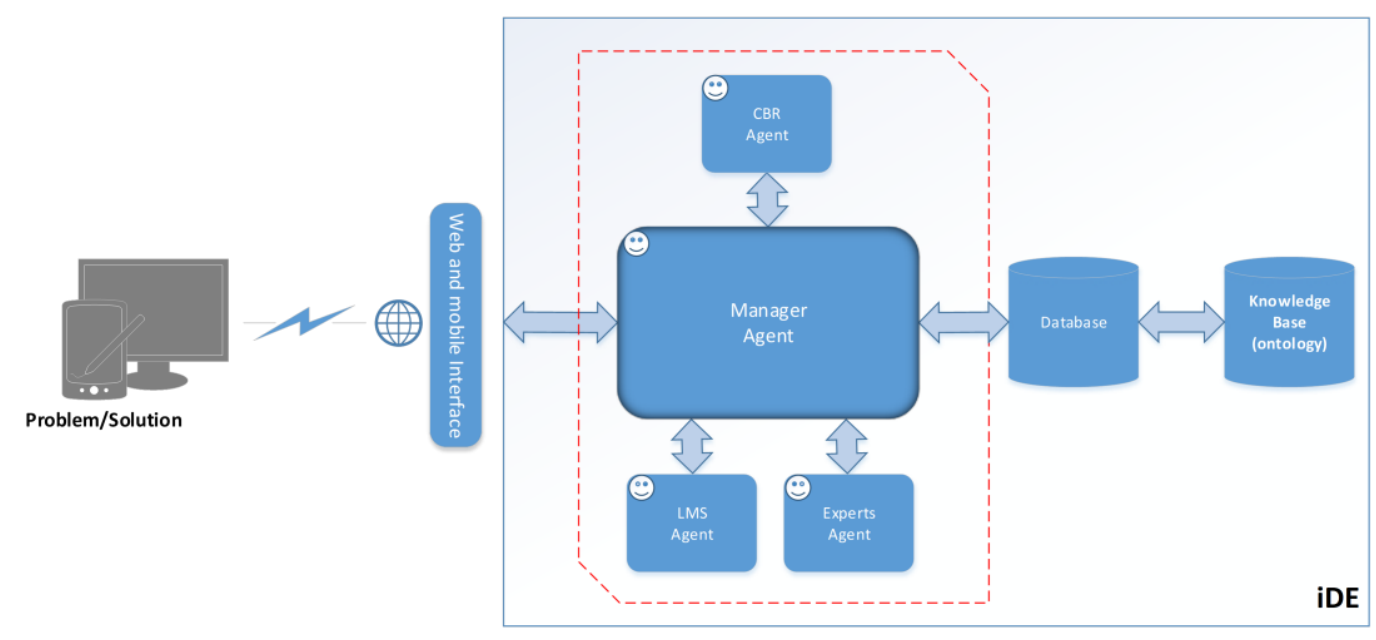

Figura 1 - Arquitetura geral do framework iDE.

- Web and Mobile interface (WM): módulo para cadastro de usuários, entrada da descrição do problema, escolha do tipo de busca de solução e combinação desejada e visualização das soluções apresentadas. Além disso, a avaliação das soluções por parte de usuários especialistas será feita também nesta interface.

- Sistema Multiagente: composto pelos agentes de software: Manager agent, CBR agent, LMS agent e Experts agent.

- Database: banco de dados que armazenará todas as informações a ser utilizada no framework iDE: os casos passados, informações do ambiente virtual e a opinião de especialistas, do que serão utilizados pelos agentes de software. Esta base de dados será acessada somente pelo Manager agent, que receberá das outras unidades as requisições de acesso aos dados.

- Knowledge base (ontologia): ontologia desenvolvida para representar o domínio do estudo de caso proposto. Formaliza os termos, entidades, relacionamentos, axiomas e instâncias usados em todo o framework iDE.

A representação do conhecimento do iDE será feita por uma ontologia modelada para formalizar o domínio do estudo de caso proposto e pela base de dados que controlará as informações que alimentarão as funções de análise e aprendizado. Essas funções serão oportunizadas pela interação do usuário com a interface, a partir de uma análise avaliativa 
VIII Congresso Brasileiro de Informática na Educação (CBIE 2019)

Anais do XXX Simpósio Brasileiro de Informática na Educação (SBIE 2019)

sobre os resultados apresentados como solução. A interface permitirá ao framework armazenar a opinião do usuário quanto à relevância das respostas através do aproveitamento ou não das mesmas como soluções de sucesso.

\subsection{As fontes de informação e o Raciocínio Baseado em Casos}

O modelo do framework iDE abrange a recuperação e tratamento de informações oriundas de três fontes: casos passados, dados provenientes de ambientes virtuais de aprendizagem e a opinião de especialistas. A partir da busca individual e da combinação destas fontes de informação, o framework oferecerá possíveis soluções para ocorrências que exijam tomadas de decisão.

O conhecimento tratado por esta técnica é armazenado na forma de casos [Wangenheim et al. 2013]. Uma vez que um caso correspondente é recuperado, o CBR adapta a solução armazenada no caso retornado para as necessidades do caso atual. A adaptação procura diferenças relevantes entre o caso recuperado e o caso atual e então aplica regras que levem essas diferenças em consideração ao sugerir uma solução.

Para o mecanismo CBR funcionar corretamente necessita-se do mapeamento de casos existentes na base. O modelo mais usado para definir o processo do CBR é o proposto por Aamodt e Plaza (1994), denominado Ciclo de CBR e composto por quatro estágios: (i) Recuperação: identifica, na base de casos, os mais similares ao problema; (ii) Reutilização: utiliza estes casos para compor a nova solução; (iii) Revisão: verifica se a solução proposta atende ao problema e (iv) Retenção: armazena a solução atual e a retém como novo caso para futura utilização em outras consultas.

Uma das grandes vantagens do uso de CBR em sistemas especialistas é a adaptação e recuperação de situações passadas em problemas atuais, e usa recursos de independência de ações [Lyra e Santos 2012]. Convém ressaltar que os novos casos não serão idênticos aos anteriores, porém os procedimentos aplicados podem ser aproveitados e reutilizados. O CBR utiliza métricas de similaridade necessárias para a etapa de recuperação do caso [Kolodner 1993]. Elas consistem em um cálculo que determina o nível de semelhança entre o caso descrito no problema e os casos anteriores, armazenados na base de casos.

As funções de análise de informações, recomendação de solução e de aprendizado do framework iDE serão realizadas pelo ciclo do CBR, conforme pode ser visto nas seções seguintes, que descreverão a forma como os agentes de software trabalharão essas estratégias para executar as ações inteligentes do arcabouço.

\subsection{Os agentes inteligentes}

O MAS do iDE é formado por quatro agentes inteligentes (ou de software) que manipulam as informações de entrada e geram as soluções adequadas a elas. Os agentes são responsáveis por executar as tarefas de busca, a análise de similaridade e as regras de aprendizado, dentro do contexto definido para cada fonte de informação. São eles:

1. Manager agent: também chamado de agente gerenciador, é o principal agente do sistema, responsável pelo gerenciamento das informações e das tarefas enviadas aos demais agentes. O Manager agent avalia as informações de entrada, repassando-as para serem avaliadas pela ontologia. As informações serão certificadas e, se forem adequadas, serão adaptadas e acrescentadas ao modelo de representação de dados e ao vocabulário ontológico. 
VIII Congresso Brasileiro de Informática na Educação (CBIE 2019)

Anais do XXX Simpósio Brasileiro de Informática na Educação (SBIE 2019)

2. CBR agent: agente que desempenha as funções da técnica de CBR. As informações de entrada são enviadas pelo Manager agent e então realiza a busca na base de casos, calculando a similaridade deles com o problema proposto.

3. LMS agent: agente responsável pela manipulação das informações oriundas dos aplicativos do LMS Moodle. Esse agente recebe as informações de entrada repassadas pelo Manager agent e executa o tratamento dessas informações, cruzando-as com os dados dos apps, a fim de calcular os níveis de similaridade. As respostas consideradas mais similares serão retornadas ao Manager agent, para preparação e envio à interface.

4. Experts agent: desempenha as funções de análise dos dados da base de opiniões dos especialistas e executa a função de similaridade desses dados com o problema repassado pelo Manager agent. As soluções mais similares são enviadas ao agente gerenciador para organização e envio à interface.

Os agentes usam a semântica formal para raciocinar sobre a associação, equivalência, consistência e classificação de classes. Sua implementação seguiu a teoria de tratamento do conhecimento abordada Ciclo do CBR.

\subsection{Funções de análise, recomendação e aprendizado}

O Manager agent, ou agente gerenciador, é o responsável pelo controle das ações dos demais agentes e da entrada, armazenamento e saída de informações do framework iDE. Ele possui funções de acesso à interface e identificação dos dados de entrada, como o problema proposto e seus elementos de caracterização. Também verifica a opção escolhida referente às fontes de informação a serem utilizadas na busca de soluções.

As tarefas de análise, recomendação e aprendizado também são controladas por esse agente e podem ser resumidas nas seguintes etapas:

- Assim que o agente os dados de entrada, é ativada a função que executa cada agente. O componente de acesso à base de dados é solicitado e os dados são lidos e enviados para o agente ativado.

- $\mathrm{O}$ agente ativado devolve uma mensagem de retorno ao Manager agent, incluindo a identificação da solução encontrada e suas informações complementares. A mensagem é decodificada e as informações são extraídas.

- Se a opção escolhida for a combinação de mais de uma fonte de informação, são ativados os agentes responsáveis pelas fontes escolhidas e enviadas as informações para eles. Quando as soluções são retornadas, o Manager agent combina-as e calcula a similaridade entre esse resultado e o problema proposto.

- Após o envio das soluções retornadas pelos agentes à interface web, serão implementadas as etapas de Reutilização, Revisão e Retenção do conhecimento junto à base de dados do framework iDE.

- Quando o Manager agent enviar à interface a solução encontrada e forem exibidas as respostas de acordo com as fontes de informação solicitadas, o usuário poderá ajudar no aprendizado do framework, verificando se a solução pode ser adaptada ao problema em questão ou não. Na interface WM, existe uma opção de avaliação das respostas sugeridas. Caso a solução recuperada seja viável ao problema proposto, os dados serão reunidos e adicionados a um registro novo na tabela de soluções relevantes do banco de dados.

Como foi adotado o Ciclo CBR para tratamento e recuperação do conhecimento no framework $\mathrm{iDE}$, todas as suas etapas foram seguidas. A etapa conhecida como 
VIII Congresso Brasileiro de Informática na Educação (CBIE 2019)

Anais do XXX Simpósio Brasileiro de Informática na Educação (SBIE 2019)

Reutilização ocorre somente após a recuperação de uma solução completa, pois é feita a reutilização das respostas retornadas como mais similares ao problema em questão.

A próxima etapa é a Revisão e acontece quando o usuário rejeita a solução. Desta forma, a solução proposta não poderá ser aplicada diretamente a um novo problema. $\mathrm{O}$ usuário analisa as diferenças entre os problemas (novo e recuperado), identifica quais partes da solução apresentada são semelhantes ao seu problema e que poderão ser aplicadas ao mesmo, vinculando assim a nova solução como resposta para a situação em questão. E a última etapa do ciclo consiste na Retenção da solução retornada como resposta possível a ser utilizada em outra situação. É o processo de armazenar o problema proposto e associá-lo à solução retornada para futuras recuperações.

\section{Estudo de caso}

Para fins de validação do framework iDE, optou-se por um estudo de caso exploratório para instanciação do arcabouço conceitual proposto. O objeto do estudo foi uma instituição que trabalha com educação a distância na oferta de cursos regulares.

O Centro de Educação a Distância da Universidade Federal do Amazonas (CED) possui atualmente cerca de 1.800 alunos (dados de 2019) distribuídos em cinco cursos de graduação oferecidos: Administração, Artes Plásticas, Biologia, Ciências Agrárias, Educação Física. O gerenciamento das atividades à distância é realizado pelo LMS Moodle (2019), onde são criadas salas de aula virtuais para as disciplinas, as quais contém o material didático, atividades de fixação e avaliativas, repositório de arquivos e espaço para visualização das notas. Esses ambientes virtuais simulam uma sala de aula física, na qual professores e alunos podem assimilar e compartilhar conteúdo, interagir entre si e, assim, construir conhecimento. Foi ainda desenvolvida uma ontologia de domínio, a ontoCED, para modelar o conhecimento tácito e explícito na abrangência pedagógica e acadêmica do CED. Ela foi desenvolvida para padronizar os termos que serão utilizados pelo framework iDE nas análises e inferências realizadas no processo de busca de soluções para os problemas propostos.

\section{Experimentos e análise dos resultados}

Os testes de funcionalidade foram realizados com dez especialistas do CED, tais como: professores, tutores e coordenadores, e que também ajudaram a compor a base de casos, em um momento anterior aos experimentos.

A base de dados disponibilizada para testes contou com: 30 casos passados reais, acontecidos no contexto do estudo de caso proposto; 30 registros de opiniões verídicas de especialistas em $\mathrm{EaD}$; e 30 gráficos com informações oriundas de aplicativos instalados no LMS Moodle, ambiente virtual utilizado no CED.

Um caso armazenado na base de casos é um registro de um problema parcialmente ou totalmente resolvido com a descrição da experiência obtida com a solução. O registro de opinião de especialista consiste em informações sobre problemas corriqueiros e de fácil solução. A Figura traz 3 um problema submetido a busca de solução por casos passados e a Figura 4, a solução apresentada pelo iDE.

Foram aplicados questionários de levantamento de qualidade pedagógica e usabilidade referentes às respostas retornadas pelo framework iDE. Os indicadores para avaliação desses requisitos podem ser observados nos seguintes resultados: as maiores notas foram dadas para os indicadores "Relevância das respostas apresentadas" e "Conhecimento agregado e feedback instrucional". Os menores valores atribuídos foram 
VIII Congresso Brasileiro de Informática na Educação (CBIE 2019)

Anais do XXX Simpósio Brasileiro de Informática na Educação (SBIE 2019)

para "Adequação das fontes de informação ao contexto pedagógico". Essas informações retratam que os entrevistados ficaram satisfeitos com o desempenho do framework no que diz respeito à relevância pedagógica das respostas e que o usuário ainda não se sente familiarizado com o uso das fontes de informação para busca de soluções.

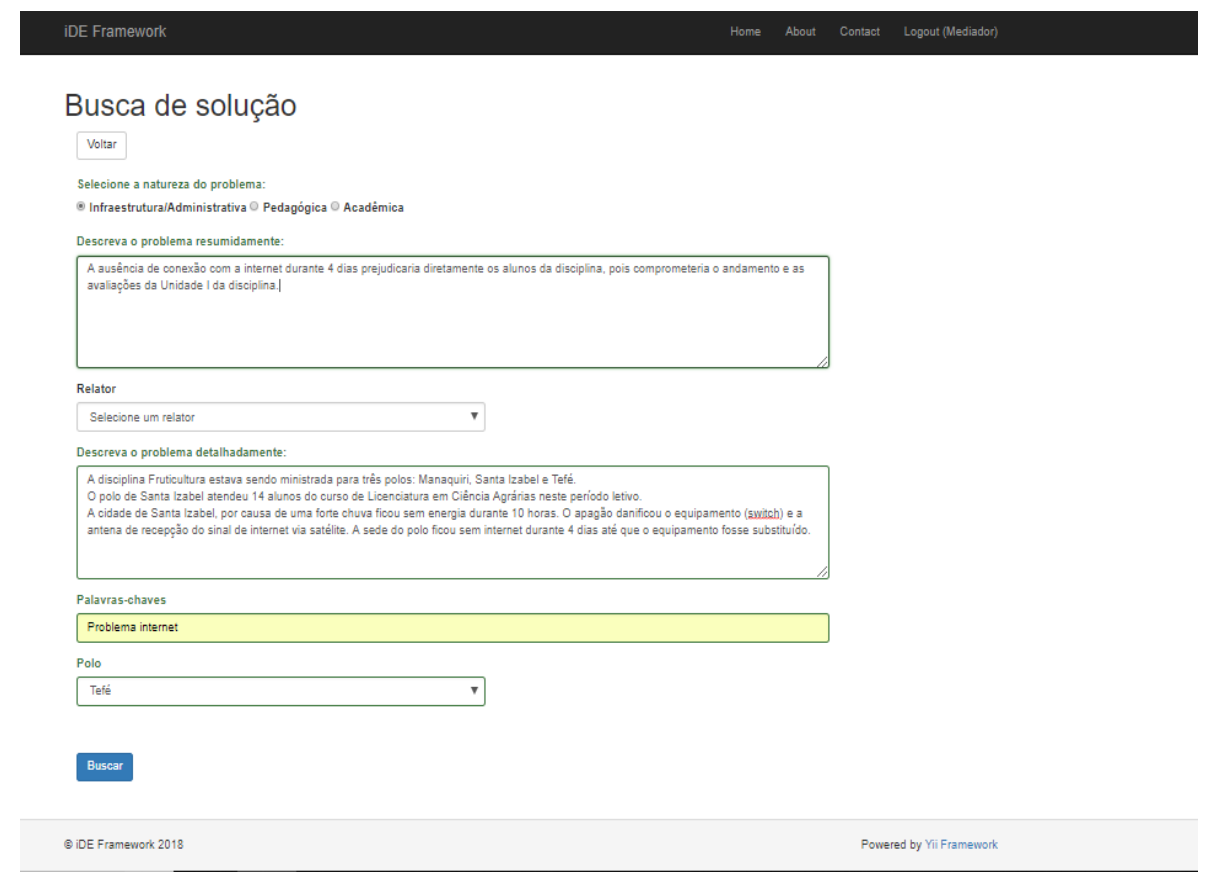

Figura 3 - Problema proposto

A segunda parte da análise das avaliações considera as respostas para a questão que ajudou a orientar nosso trabalho: "O conhecimento recuperado apresenta soluções relevantes para a resolução de problemas frequentes em ambientes de $\mathrm{EaD}$, a partir de sua aplicação no contexto do estudo de caso proposto?"

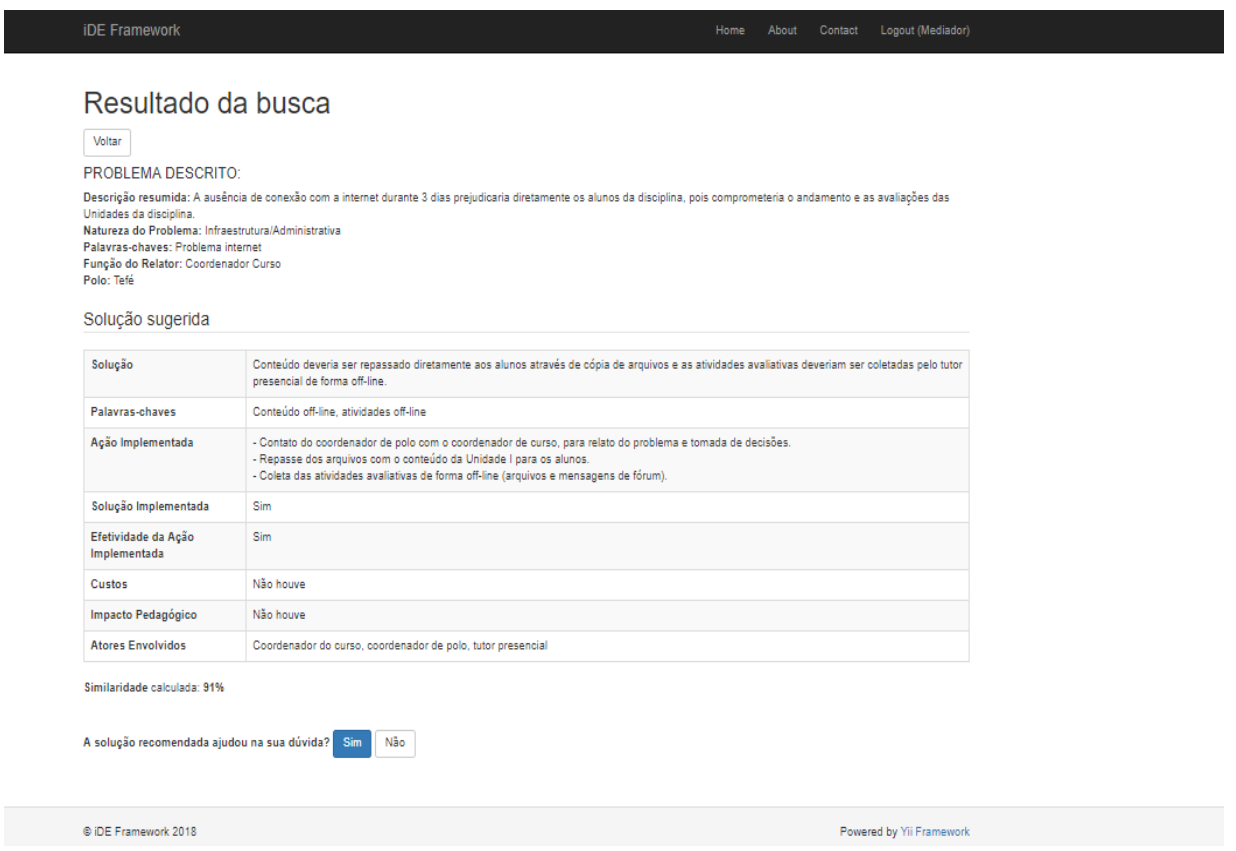

Figura 4 - Resultado da busca por casos passados 
VIII Congresso Brasileiro de Informática na Educação (CBIE 2019)

Anais do XXX Simpósio Brasileiro de Informática na Educação (SBIE 2019)

Os comentários de análise serão balizados pelo gráfico da Figura 5:

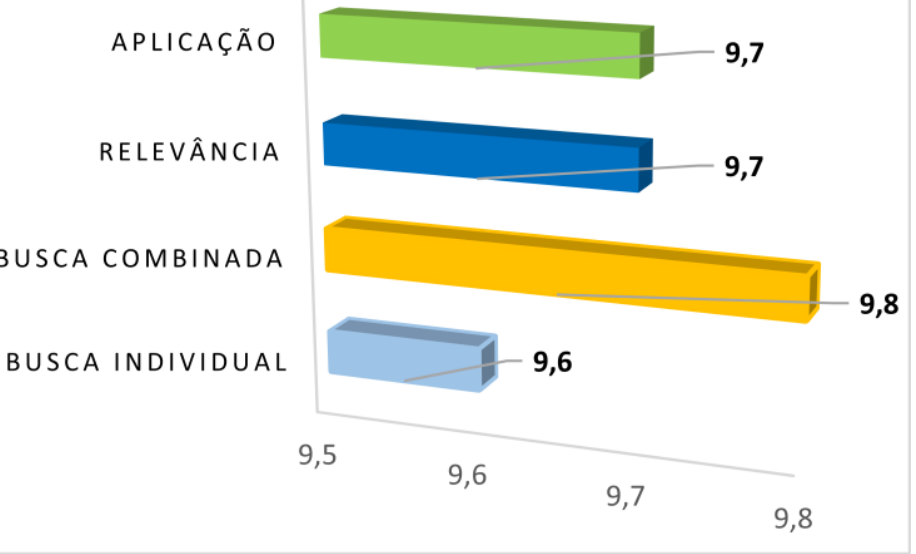

Figura 5 - Gráfico: Retorno sobre soluções apresentadas.

Nos quesitos "Relevância" e "Aplicação" das soluções encontradas, as médias das notas atribuídas tenderam a um mesmo valor $(9,7)$, isso traduz a opinião positiva sobre a importância das soluções retornadas pelo arcabouço e a sua aplicabilidade prática no cotidiano de cursos oferecidos à distância.

Percebe-se pelo gráfico que a busca de solução para o problema proposto utilizando somente uma fonte de informação obteve valores menores que a busca combinando várias fontes de informação. Ou seja, na opinião dos entrevistados, a combinação das buscas melhorou em cerca de $2 \%$ a qualidade da solução apresentada.

\section{Conclusão}

Um dos principais desafios das instituições que promovem cursos na modalidade à distância é acompanhar o desempenho acadêmico do aluno e disponibilizar infraestrutura física e pedagógica adequada ao curso oferecido. Além disso, também compõem esse obstáculo o reaproveitamento do conhecimento tácito, representado pela experiência de especialistas locais, que quase sempre é utilizado para solucionar questões recorrentes em localidades com problemas característicos da Região Amazônica.

Para tanto, este artigo apresentou o iDE, um framework que utilizou diferentes fontes de informação e suas possíveis combinações com o objetivo de mapear o conhecimento implícito em informações oriundas de situações antigas e equivalentes a um problema proposto, a fim oferecer soluções que possam ser consideradas relevantes para resolvê-lo. O iDE usou as técnicas de Raciocínio Baseado em Casos e de Sistemas Multiagentes para extrair as informações que foram combinadas para gerar respostas a um problema dado como entrada. A relevâncias das soluções obtidas foram avaliadas por especialistas na área, por meio de uma interface web, o que contribuiu para o aprendizado do arcabouço.

As técnicas de IA aqui utilizadas apresentaram-se como fundamentais para a recuperação e manipulação os conhecimentos explícitos e implícitos, segundo a opinião dos entrevistados. As funções dos agentes de software tornaram possível $o$ reaproveitamento de sugestões anteriores para compor novas soluções para problemáticas parecidas. A recuperação de soluções anteriores e o seu reaproveitamento em problemas novos apresentou-se como uma boa estratégia para solução de dificuldades comuns aos cursos à distância, na opinião de usuários especialistas. 
VIII Congresso Brasileiro de Informática na Educação (CBIE 2019)

Anais do XXX Simpósio Brasileiro de Informática na Educação (SBIE 2019)

Desta forma, os resultados obtidos potencializaram o conhecimento baseado em experiências anteriores para solucionar novos problemas. As respostas indicam sugestão de ações interventivas, que são as soluções propostas pelas informações recuperadas e que podem ser aplicadas por mediadores de cursos à distância. A partir dos experimentos com o framework pode-se perceber que, em seu domínio, o conhecimento é continuamente convertido e criado à medida que os usuários utilizam, colaboram, interagem e aprendem. Assim, conclui-se ser uma alternativa factível o uso do framework iDE para orientar a resolução de problemas a partir de situações anteriores e semelhantes.

\section{Referências}

Aamodt, A. and Plaza, E. (1994) "Case-based reasoning: Foundational issues, methodological variations, and system approaches". AI Com. v.7, n.1, pp. 39-59.

Bittencourt, I., Tadeu, M. and Costa, E. (2006) "Combining AI Techniques into a Legal Agent-based Intelligent Tutoring Systems". In: Proceedings of $18^{\text {th }}$ Int. Conf. on Software Engineering and Knowledge Engineering. San Francisco, USA.

Bremgartner, V., Netto, J. F. M. and Menezes, C. (2014) "Using Agents and Open Learner Model Ontology for Providing Constructive Adaptative Techniques in Virtual Learning Enviroments". In: Proceedings of 14th Ibero-American Conference on Artificial Intelligence (IBERAMIA), pp. 625-636. Santiago, Chile.

Garrido, J. L., Hurtado, M. V., Noguera, M. and Zurita, J. M. (2008) "Using a CBR approach based on ontologies for recommendation and reuse of knowledge sharing in decision making". In: Proceedings of the 8th International Conference on Hybrid Intelligent Systems. IEEE 2008. Barcelona, Spain.

Kolodner, J. L. (1993) “Case-Based Reasoning”. Morgan Kaufmann Pub., Inc.

Lyra, F. e Santos, N. (2012) "Agentes de Software no Monitoramento de Alunos em Educação a Distância”. In: I Congresso Brasileiro de Informática na Educação. In: Anais do XVIII Workshop de informática na Escola, Rio de Janeiro, Brasil.

Mendes, W., Girardi, R. e Leite, A. (2013) "Arquitetura baseada em ontologias de um agente RBC". In: Information Systems and Techologies (CISTI), 2013. 8th Iberian Conference on. Lisboa.

Moodle. (2019) "Modular Object-Oriented Dynamic Learning Environment". http://moodle.org/. Acessado em 05/06/2019.

Silva, J. S., Teles Lucena, K. K., Oliveira, E. H. T. de. (2015) "WebMonitor: uma ferramenta para monitoramento e acompanhamento de cursos em um AVA". In: XXVI Simpósio Brasileiro de Informática na Educação. In: Anais do IV Congresso Brasileiro de Informática na Educação (CBIE), p. 249-258. Maceió, Brasil.

Teles Lucena, K. K., Gusmão, T. H. Y., Abreu, A. e Oliveira, E. H. T. de. (2017) "Uma arquitetura de agente RBC baseada em ontologias para suporte a EaD". In: XXVIII Simpósio Brasileiro de Informática na Educação. In: Anais do VI Congresso Brasileiro de Informática na Educação (CBIE), p. 1537-1546. Recife, Brasil.

Teles Lucena, K. K. (2018) "iDE - um framework para suporte ao gerenciamento de cursos em Educação a Distância”. Tese de Doutorado. Instituto de Computação, Universidade Federal do Amazonas, Brasil.

Wangenheim, C. G. V., Wangenheim, A. V. e Rateke, T. (2013) "Raciocínio baseado em casos - com software livre e aplicativos móveis". $2^{\mathrm{a}}$ edição atualizada. Florianópolis: Editora Bookness. 